\title{
IDENTIFICACION DEL CILINDRO NUDOSO EN IMÁGENES TC DE TROZAS PODADAS DE PINUS RADIATA UTILIZANDO REDES NEURONALES ARTIFICIALES
}

\section{IDENTIFICATION OF KNOTTY CORE IN PINUS RADIATA LOGS FROM COMPUTED TOMOGRAPHY IMAGES USING ARTIFICIAL NEURAL NETWORK}

Gerson Rojas-Espinoza ${ }^{1}$, Oscar Ortíz-Iribarren²

\section{RESUMEN}

La factibilidad de identificar el cilindro nudoso en imágenes de tomografía computarizada de rayos X (TC) de trozas podadas de pino radiata (Pinus radiata $\mathrm{D}$. Don), fue evaluada utilizando un método de clasificación supervisada basado en Redes Neuronales Artificiales (RNA). El proceso de clasificación consideró también la identificación de la zona libre de defectos y nudos. Treinta trozas podadas de pino radiata fueron escaneadas en un escáner médico multi-slice de rayos X, donde las imágenes TC resultantes fueron obtenidas cada $5 \mathrm{~mm}$. Un total de 270 imágenes TC fueron clasificadas utilizando la técnica Redes Neuronal Artificial y los mapas temáticos resultantes, fueron filtrados con un filtro de mediana de 7 x 7 . La precisión del proceso de clasificación de las imágenes TC fue obtenida a partir de una matriz de confusión y el estadístico Kappa. Los resultados indicaron que el cilindro nudoso puede ser identificado y separado con una precisión de $92.7 \%$, mientras que para la precisión global se obtuvo un valor de $85.0 \%$. Tras filtrar los mapas temáticos, los valores de precisión aumentaron a $96.3 \%$ y $92.3 \%$ para el cilindro nudoso y la precisión global, respectivamente. Los valores Kappa fueron de 0.607 y 0.764 para los mapas temáticos y mapas temáticos filtrados, respectivamente. Estos valores indicaron que existe un fuerte grado de conformidad entre los datos de referencia y el proceso de clasificación. Los resultados sugieren que es factible aplicar RNA como procedimiento de clasificación para identificar el cilindro nudoso en imágenes TC de trozas podadas de pino radiata.

Palabras claves: Cilindro nudoso, tomografía computarizada, redes neuronales artificiales, matriz de confusión, pino radiata

\section{ABSTRACT}

The feasibility of identifying Knotty core in images of X-ray computed tomography (CT) of pruned radiata pine logs (Pinus radiata D. Don), was evaluated using a supervised classification method based on artificial neural networks (ANN). The classification process also considers the identification of the clear wood and knots. Thirty pruned radiata pine logs were scanned in a multislice scanner medical X-ray, where the resulting CT images were obtained every $5 \mathrm{~mm}$. A total of $270 \mathrm{CT}$ images were classified using the ANN, and the resulting thematic maps were filtered with a median filter of $7 \times 7$. The accuracy of the classification process of the CT images was obtained from a confusion matrix and Kappa statistics. The results indicated that the Knotty core can be identified and separated with an accuracy of $92.7 \%$, while for the overall accuracy was obtained a value of $85.0 \%$. After filtering thematic maps, the precision values increased to $96.3 \%$ and $92.3 \%$ for the defective core and overall accuracy, respectively. Kappa values were 0.607 and 0.764 for thematic maps and thematic maps filtered, respectively. These values indicate that there is a strong degree of agreement between reference data and classification process. The results suggest that it is feasible to apply artificial neural networks as classification procedure to identify the Knotty core in CT images of pruned radiata pine logs.

Keywords: Knotty core, computed tomography, artificial neural networks, confusion matrix, radiata pine. 


\section{INTRODUCCION}

En general, la eficiencia del proceso de aserrío se ve influenciada principalmente por la calidad de la materia prima, donde la presencia de defectos externos e internos en las trozas tiene un efecto significativo sobre la calidad final de la madera aserrada producida. La presencia de defectos en la madera tales como nudos, grietas internas, bolsas de resina, bolsas de corteza, entre otros, castiga drásticamente el precio final de la madera. En Chile, las principales empresas madereras realizan grandes esfuerzos por mejorar la calidad de sus bosques, principalmente, de la especie pino radiata, la cual sustenta la industria maderera debido a su disponibilidad y variadas aplicaciones de su madera. En esta dirección, la poda es el principal tratamiento silvícola que las empresas están aplicando a los bosques de pino radiata para obtener fustes de mejor calidad. Este tratamiento silvícola, es una actividad que elimina las ramas vivas o muertas del árbol y permite la producción de madera libre de nudos, una vez que el crecimiento en diámetro del fuste cubre la zona de defecto. De esta manera, la parte interna de las ramas podadas queda confinada en una zona central, respecto al volumen del fuste, zona denomina cilindro nudoso $(\mathrm{CN})$. El diámetro del $\mathrm{CN}$ depende principalmente de la calidad del sitio, el número de podas y edad del árbol en el momento de la poda. Así, la identificación del $\mathrm{CN}$ antes de iniciar el proceso de aserrío se transforma en uno de los principales problemas de los aserraderos. Normalmente los operadores utilizan las características de la superficie de la troza como indicadores para predecir la localización y tipo de defectos internos

En general, la identificación de las características y defectos internos de las trozas requiere de la utilización de técnicas no destructivas. En este sentido, diferentes técnicas, algunas de ellas habitualmente utilizadas en la ciencia médica como una herramienta para examinar y evaluar de manera no destructiva la estructura del cuerpo humano, han sido evaluadas con el objetivo de caracterizar internamente las trozas. Los ultrasonidos (Birkeland y Han 1991), la resonancia magnética nuclear (Chang et al. 1989; Coates et al. 1998) y Radiación gamma (Hagman 1993, Karsulovic et al. 2002 y 2005) son algunas de las técnicas utilizadas para este fin. Sin embargo, la Tomografía Computarizada (TC) de rayos X, ha mostrado un gran potencial para detectar defectos internos y otras irregularidades en trozos de coníferas y latifoliadas (Taylor et al. 1984; Funt y Bryant 1987; Zhu et al. 1991; Li et al. 1996; Guddanti y Chang 1998; Bhandarkar et al. 1999; Schmoldt et al. 1995, 1998, 2000 and Nordmark 2002, 2003).

Taylor et al. (1984) evaluaron un sistema automático de segmentación de imágenes TC, basado en el nivel de gris obtenido a partir de histogramas de intensidad de frecuencia, para identificar y localizar nudos. Funt y Bryant (1987) desarrollaron un programa que interpreta automáticamente las imágenes TC y que utiliza la alta densidad y la forma elíptica de los nudos y la baja densidad, junto con la textura rugosa de zonas con pudrición, para diferenciar la madera podrida de la madera libre de defectos. Estos autores coinciden en que la variación del contenido de humedad en la troza en algunos casos dificulta la identificación. Schmoldt et al. (1995) indicaron que el nivel de gris de las imágenes TC está directamente relacionado con la densidad, variando dramáticamente con las diferentes especies y contenido de humedad. Oja y Temnerud (1999) reportaron que en imágenes TC de trozas verdes de Abeto Rojo (Picea abies (I.) Kars), el nivel de gris de la madera difiere mucho entre duramen y albura, debido principalmente a la diferencia en el contenido de humedad. Una situación similar fue observada en imágenes TC de trozas de Arce (Acer saccharum Marsh) por Rojas et al. (2005).

En otra dirección, varios métodos de clasificación de imágenes TC, basados en el conocimiento a priori del nivel de gris asociado a cada característica o defecto interno han sido evaluados, con el fin de mejorar la capacidad de interpretación de este tipo de imágenes. Rojas et al. $(2006,2007)$ presentaron un método de clasificación supervisada basado en el algoritmo de máxima verosimilitud (CMV), para identificar y separar la albura del duramen, nudos y pudrición, a partir de imágenes TC de trozas de Arce (Acer saccharum Marsh). Los resultados indicaron que la albura puede ser identificada con una precisión de $97.8 \%$, mientras que la precisión global fue de $82.6 \%$. Por su parte, para esta especie y utilizando el algoritmo (CMV) y un análisis de textura de las imágenes TC, 
Wei et al. (2008a) reportaron valores de precisión de $85.6 \%, 72.1 \%$ y 83\% para la detección de la albura, nudos y corteza respectivamente. Recientemente, Rojas y Ortiz (2009) utilizaron el CMV para identificar y separar el CN en imágenes TC de trozas podadas de pino radiata. De acuerdo a estos autores, el CN puede ser separado con una precisión de $98.5 \%$.

En esta dirección, la utilización de algoritmos de clasificación basados en redes neuronales artificiales (RNA) también ha sido evaluada. Schmoldt et al. (2000) utilizaron RNA para la identificación y clasificación de albura, corteza, nudos y grietas en roble rojo (Quercus rubra L.), álamo amarillo (Liriodendron tulipifera L.) y cerezo negro (Prumus serotina Ehrh). Si bien estas características internas fueron identificadas en las distintas especies, al igual que en los estudios citados anteriormente, existía superposición entre el nivel de gris asociado a las diferentes características o defectos internos. Nordmark (2002) también utilizó RNA, para identificar nudos en trozas de pino silvestre (Pinus sylvestris L.). Si bien los nudos pudieron ser separados del resto de la madera con una precisión de $95.9 \%$, existe un cierto grado de superposición del nivel de gris en la zona límite entre los nudos y la madera libre de defectos. De igual manera, este autor utilizó esta técnica para desarrollar modelos para predecir nudos y la geometría de las trozas de pino silvestre (Nordmark2003), reportando un valor de precisión de 90\%. En esta dirección, Wei et al. (2008b) desarrollaron un método que utiliza RNA y análisis textural para identificar albura duramen, nudos y corteza en imágenes TC de trozas de arce (Acer saccharum Marsh) y picea negra (Picea mariana (Mill)). Los resultados de este estudio indicaron que una precisión de global de $89.5 \%$ y $92.4 \%$ puede ser obtenida para arce y picea negra, respectivamente.

En general, de acuerdo a la literatura revisada, queda de manifiesto que la tomografía computarizada de rayos $\mathrm{X}$, es un método no destructivo que permite identificar los defectos y características internas de las trozas. Sin embargo, el problema no está completamente resuelto. La compleja estructura y variabilidad inherente a la madera, las variaciones en la densidad y contenido de humedad limitan, en algunos casos, la precisión en la identificación de los defectos internos. En este sentido el objetivo principal de este estudio es evaluar la factibilidad de utilizar redes neuronales artificiales para identificar el cilindro nudoso en imágenes TC de trozas podadas de pino radiata.

\section{MATERIALES Y METODOS}

\section{Materiales.}

Treinta árboles podados de pino radiata (Pinus radiata D. Don) de 23 años de edad, fueron seleccionados y talados en el predio El Espigado, localizado en la zona de Arauco, distante a $100 \mathrm{Km}$ de la ciudad de Concepción. La primera poda de estos árboles fue realizada a los 5 años de edad y a una altura de $2.7 \mathrm{~m}$. De cada árbol se obtuvo una troza de la zona podada del árbol, de largo comercial equivalente a $4.3 \mathrm{~m}$ aproximadamente y enviados para su inmediato procesamiento en el pabellón de tecnología de la madera de la Universidad del Bío-Bío. Cada rollizo fue trozado en tres partes, de un metro de longitud cada una. Las trozas obtenidas fueron marcadas y luego selladas en cada extremo con impermeabilizante Anchorseal, para evitar la pérdida de humedad. Un total de noventa trozas fueron obtenidas y utilizadas como material de ensayo.

Las trozas fueron escaneadas en un escáner médico de rayos X (Tomografía computarizada (CT)), de marca Philips, multi-Slice, perteneciente al laboratorio de imagenología del Hospital del Trabajador, en Concepción. El proceso de escaneo fue realizado a $120 \mathrm{kVp}$ y $249 \mathrm{~mA}$, donde las imágenes TC resultantes fueron obtenidas cada $5 \mathrm{~mm}$, en formato de 512 x 512 píxeles y con resolución de 8 bits. La resolución espacial de un píxel, varió entre 0.7 y $0.9 \mathrm{~mm}$, dependiendo del diámetro de cada troza. Luego, tres imágenes TC fueron seleccionadas por cada troza, obteniendo finalmente una muestra de 270 imágenes, las que fueron analizadas en este estudio. 


\section{Clasificación de imágenes TC usando redes neuronales artificiales}

Las RNA son modelos computacionales que se desarrollaron originalmente para modelar la forma en que el cerebro humano desarrolla una determinada tarea y actualmente se aplican en la clasificación de imágenes (reconocimiento de patrones) y otros trabajos de análisis de imágenes (Wei et al. 2008b). Estos modelos o sistemas de procesamiento de la información consisten en un gran número de elementos simples llamados nodos o neuronas que están organizados en capas. Cada neurona está conectada con otras neuronas mediante enlaces de comunicación, cada uno de los cuales tiene asociado un peso. En los pesos se encuentra el conocimiento que tiene la RNA acerca de un determinado problema (Palmer et al. 2000)

La RNA generalmente consta de una capa de entrada, una o más capas ocultas y una capa de salida. La primera etapa para desarrollar un RNA consiste es definir un grupo de entrenamiento, que corresponde a grupos de píxeles asociado a cada patrón o clase a identificar. Estos grupos de píxeles se obtienen directamente de un grupo de imágenes seleccionadas para este fin. La segunda etapa consiste en entrenar la RNA con el grupo de entrenamiento obtenido. Durante este proceso de aprendizaje de la RNA, los pesos son modificados de forma iterativa de acuerdo con los valores del grupo de entrenamiento. En esta etapa se deben definir los valores de la tasa de aprendizaje y el momento, variables importantes para el entrenamiento de la RNA, ya que controlan el tamaño del cambio de los pesos en cada iteración (Palmer et al. 2000). La tasa de aprendizaje es la responsable de acelerar el proceso de aprendizaje o velocidad de respuesta de la RNA. A mayor tasa de aprendizaje, mayor es la modificación de los pesos en cada iteración y por ende el aprendizaje será más rápido, pero por otro lado puede ocasionar oscilaciones. Si el valor es demasiado bajo, el sistema tomará mucho tiempo para converger a la solución. El momento se utiliza para controlar las posibles oscilaciones o inestabilidades que se generan en la variación de los pesos y reduce la posibilidad que el sistema converja hacia un mínimo local. La expresión matemática de la regla de modificación de los pesos se indica a continuación (Rumelhart et al. 1986):

$$
\Delta W_{j i}(n+1)=\varepsilon \delta_{p j} x_{p i}+\eta \Delta W_{j i}(n)
$$

Donde $W_{j i}$ es el peso entre la neurona $i$ y la neurona $j, n$ es el número de la iteración, $\varepsilon$ corresponde a la tasa de aprendizaje, $\delta_{p j}$ es el error de la neurona $j$ para el patrón $p, x_{p i}$ es la salida de la neurona $i$ para el patrón $p$ y $\eta$ corresponde al momento.

Entre los diversos tipos de RNA, la configuración retro propagación (back-propagation (BP) $\mathrm{ANN}$ ) ha sido la más popular, ya que son muy eficaces en el reconocimiento de patrones y con una implementación bastante simple (Schmoldt et al. 2000). Este tipo de RNA ha sido utilizado en el presente estudio.

El procedimiento de análisis de las imágenes se inició con la generación del grupo de entrenamiento, obtenido a partir de una muestra de píxeles de las clases o patrones a identificar, en este caso, CN, madera libre de defectos (MLD) y nudos. Este grupo de entrenamiento fue utilizado como información de entrada por el sistema de redes neuronales, el cual posteriormente realiza el proceso de clasificación. Valores de 0.15 y 0.5 fueron utilizados para la tasa de aprendizaje y momento, respectivamente, con un total de 500 iteraciones. Otros valores de tasa de aprendizaje y momentun fueron evaluados, sin embargo los utilizados presentaron mejor desempeño. Finalmente, se llevó a cabo la clasificación de las imágenes TC, en donde se tenía una capa de entrada y una capa de salida con tres nodos de salida correspondiente a las tres características de las trozas de Pinus radiata que se deseaban identificar. Luego de la clasificación, los mapas temáticos fueron filtrados utilizando un filtro de mediana de $7 \times 7$ con el objetivo de eliminar el ruido generado por el proceso de clasificación.

Finalmente, para evaluar cuantitativamente este procedimiento de clasificación de imágenes TC, valores de precisión de detección del CN, MLD y nudos, fueron obtenidos a partir de la matriz 
de confusión determinada para cada imagen TC evaluada (Rojas y Ortiz 2009). En este método, la precisión fue determinada mediante la comparación entre los píxeles de la imagen que fueron correctamente clasificados y los mismos píxeles en la imagen sin clasificar (imagen de referencia ó imagen real). El resultado de esta comparación es expresado en la forma de una matriz de confusión o de error. En esta matriz, los datos de referencia (representados por las columnas de la matriz) son comparados con los datos clasificados (representados por las filas de la matriz). La diagonal principal indica el grado de acuerdo entre ambos grupos de datos. La precisión (expresada en porcentaje) para cada clase fue determinada a partir de la matriz de confusión, dividiendo el número de píxeles correctamente clasificados por el número total de píxeles en los datos de referencia. La precisión global (incluye todas las clases) para una imagen clasificada fue también calculada dividiendo en total de píxeles correctamente clasificados (suma de la diagonal principal) por el numero total de píxeles en la matriz de confusión (Story y Congalton 1986). Un valor de 100 para los valores de precisión global indica un acuerdo perfecto entre los resultados de la clasificación y los datos de referencia. Al mismo tiempo, los errores de omisión y comisión pueden ser también determinados a partir de la matriz de confusión. Los errores de omisión corresponden a píxeles que pertenecen a una clase de interés y que han sido clasificados erróneamente en otra clase, mientras que los errores de comisión corresponden a píxeles de otras clases y que el clasificador ha asignado a una clase de interés.

El estadístico Kappa fue también determinado como complemento a los valores de precisión obtenidos de la matriz de confusión. Éste índice estadístico mide el grado de ajuste, exclusivamente para la predicción de la clasificación y utiliza para el análisis los resultados de la matriz de confusión (Jensen 1996). El estadístico K es calculado como sigue :

$$
K=\frac{N \sum_{i=1}^{r} x_{i i}-\sum_{i=1}^{r}\left(x_{i+} *^{2} x_{+i}\right)}{N^{2}-\sum_{i=1}^{r}\left(x_{i+} *^{*} x_{+i}\right)}
$$

donde $r$ es el numero de filas en la matriz, $x_{i i}$ es el numero de píxeles en la fila $i$ y columna $i, x_{i^{+}}$y $x_{+i}$ corresponden al total marginal para la fila y y columna i, respectivamente, y $N$ es el numero total de píxeles.

Para este estudio, se utilizaron los 270 mapas temáticos del proceso de clasificación. La matriz de confusión asociada a cada mapa temático fue obtenida a partir de una muestra de 70 píxeles seleccionados aleatoriamente (Richards y Jia 1999) de cada mapa temático. Este análisis también fue realizado para cada mapa temático filtrado, utilizando exactamente los mismos 70 píxeles para cada uno de los mapas temáticos. El procedimiento de clasificación RNA y la evaluación cuantitativa fueron realizados utilizando el programa computacional PCI versión 10.0 (PCI 2005).

\section{RESULTADOS Y DISCUSION}

\section{Escaneo de trozas}

Un ejemplo típico del resultado del proceso de escaneo y que corresponde a una imagen TC de una sección transversal de una troza podada de pino radiata, se presenta en la fig. 1a. Por su parte, la fig. 1b muestra la fotografía digital de la sección transversal asociada a la imagen TC presentada en la fig. 1a. En general, en la fig. 1a se observan claramente las características y defectos internos de la troza tales como anillos de crecimiento, CN, MLD y nudos. Se puede observar también la zona de madera de primavera y de verano que forma parte de los anillos de crecimiento, las cuales presentan mayores anchos en la zona del CN que en MLD. La madera libre de defectos presenta un mayor nivel de gris (escala de gris) que el interior del CN. Los nudos al interior del CN, se observan claramente debido a su forma y mayor nivel de gris. 


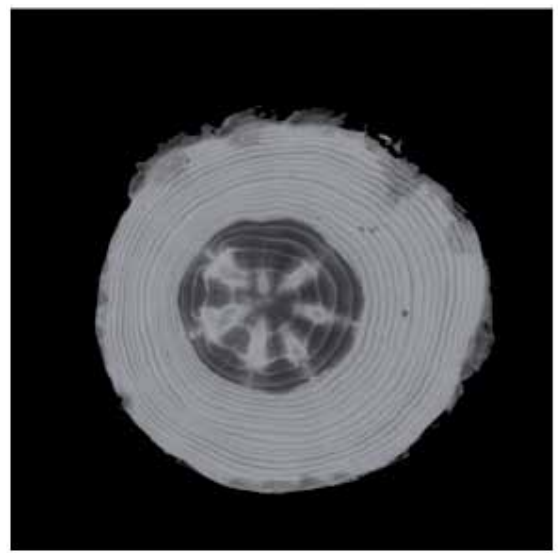

(a)

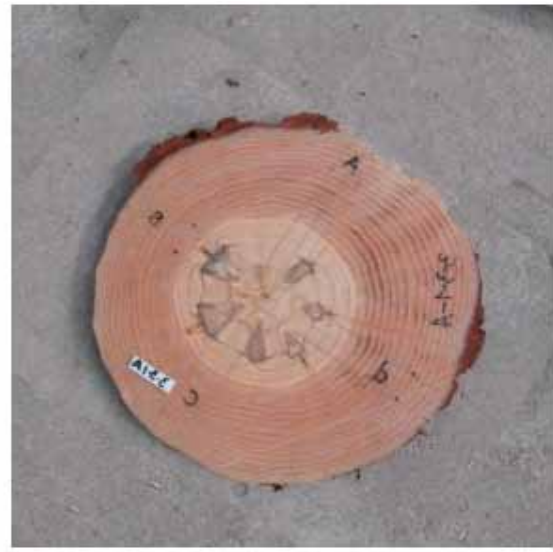

(b)

Figura 1. (a) Imagen TC original de una sección transversal de una troza de pino radiata podada. (b) Imagen real digital correspondiente a la imagen TC original.

Al comparar ambas figuras, se observa que la imagen TC (Fig.1a ) es una copia fiel de la fotografía digital (Fig. 1b). Esto ratifica lo reportado en la literatura para otras especies de madera, en el sentido que la Tomografía Computarizada presenta un gran potencial para la evaluación interna de trozas y en particular para la especie pino radiata, objeto de evaluación en el presente estudio.

\section{Clasificación de imágenes TC utilizando Redes Neuronales Artificiales}

Los resultados obtenidos del proceso de clasificación y post clasificación de imágenes TC utilizando RNA se presentan en las figuras $2 \mathrm{a}$ y $2 \mathrm{~b}$, respectivamente. La fig. 2a, muestra el mapa temático (imagen TC clasificada) obtenido de la clasificación con RNA, considerando tres clases a identificar, esto es, CN, MLD y nudos. La fig. $2 b$ muestra el resultado de la aplicación de un filtro de mediana 7x7 al mapa temático presentado en la fig. 2a. En general, en la fig. 2a se observa que el $\mathrm{CN}$ (color azul) puede ser identificado y separado de MLD (color amarillo). Sin embargo, se observa una superposición del nivel de gris asociado a los nudos presentes en el CN y con la zona MLD. Esta situación se debe principalmente a la diferencia en la densidad de la madera de primavera y verano presente en los anillos de crecimiento (Kininmonth y Whitehouse 1991) y también a la variación del contenido de humedad en el interior de los nudos. Algo similar ocurre en la periféria de la troza, donde también se observa una superposición del nivel de gris, que puede ser atribuido a la pérdida de humedad debido a la ausencia de corteza. En general, la variación del contenido de humedad en el interior de las trozas en algunos casos dificulta la identificación, situación que ha sido reportada anteriormente por otros autores. Según (Oja 2000) el nivel de gris en todas las áreas de la sección transversal de la troza se ve afectada por la variación del contenido de humedad. En este sentido, Rojas et al. $(2005,2007)$ indicaron que la densidad verde es la propiedad física que mejor se correlaciona con el nivel de gris de las imágenes TC.

En general, el problema de la variación del nivel de gris al interior de los anillos de crecimiento debido a la diferencia en la densidad de la madera de primavera y de verano, es eliminado totalmente después de aplicar un filtro de mediana de $7 \times 7$ a los mapas temáticos (Fig. 2b). En esta figura, se observa también que el ruido generado en la periferia de la troza, asociado principalmente a una disminución del contenido de humedad, es eliminado en forma muy leve. Por otra parte en el interior del cilindro nudoso no se observan mayores cambios, al igual que en la zona interna de los nudos, por lo que persiste la superposición de nivel de gris. 


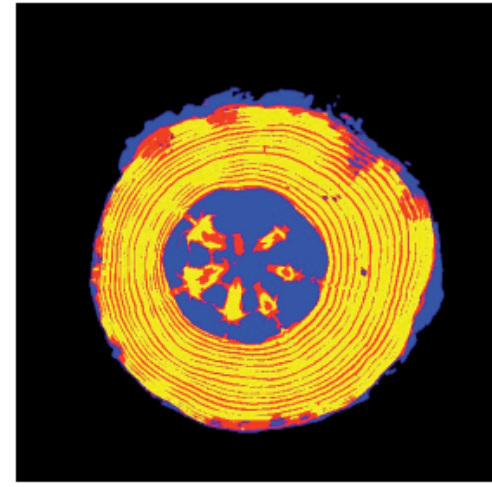

(a)

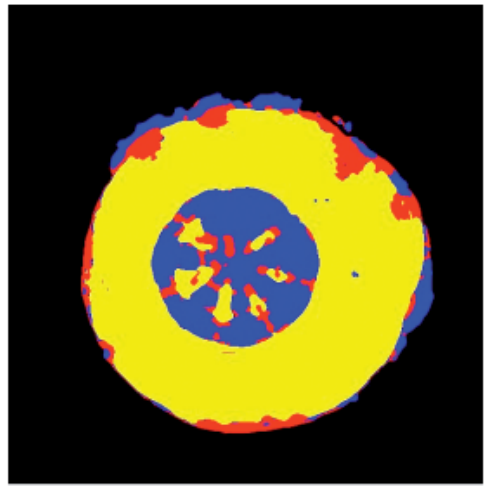

(b)

Figura 2. (a) Mapa temático obtenido de la clasificación utilizando RNA.

(b) Mapa temático filtrado utilizando un filtro de mediana de 7x7.

\section{Evaluación de la precisión}

Los resultados del análisis cualitativo de los mapas temáticos indicaron que el CN puede ser identificado utilizando el clasificador basado en RNA. Sin embargo, esto no es suficiente para obtener conclusiones más robustas. Por esta razón, a partir de la determinación de la matriz de confusión asociada a cada mapa temático es posible obtener valores de precisión de la capacidad de identificación del CN, MLD y nudos.

La tabla 1 muestra la matriz de confusión obtenida a partir de la evaluación de 270 mapas temáticos. En esta matriz se presentan los valores de precisión individual y también los valores de precisión global. Los datos contenidos en esta matriz corresponden a número de píxeles. Estos datos pueden ser utilizados también para calcular los errores de omisión y comisión ocurridos durante el proceso de clasificación de las imágenes TC. Sin embargo en este estudio solo interesan los errores de omisión. A partir de esta tabla se tiene que la precisión de detección de CN, MLD y nudos fue de $92.7 \%, 84.8 \%$ y $52.8 \%$, respectivamente, mientras que para la precisión global se obtuvo un valor de $85.0 \%$. En general, estos valores individuales de precisión pueden ser explicados por el error de omisión, el cual permite identificar las clases a las cuales los píxeles fueron asignados. Por ejemplo, el error de omisión para el CN fue de $7.3 \%$ (151/2078), esto significa que 151 píxeles de CN fueron erróneamente clasificados en las clases nudos (146 píxeles) y MLD (5 píxeles). En otras palabras, existe una leve superposición del nivel de gris particularmente entre $\mathrm{CN}$ y nudos, equivalente a un $7 \%$. En esta dirección, para MLD se obtuvo un error de omisión de $15.2 \%$, del cual el 13.3\% (1.607/12.086) está asociado a la clase nudos y el 1.9\% (226/12.086) al CN. En este caso, la superposición del nivel de gris está principalmente asociada a la clase nudos. De la misma manera, un error de omisión de $47.2 \%$ fue obtenido para la clase nudos, el cual está asociado casi en su totalidad a la clase MLD (46.6\%).

Tabla 1. Matriz de confusión obtenida para los mapas temáticos.

\begin{tabular}{|lcccc|}
\hline & \multicolumn{4}{c|}{ Píxeles en clases verdaderas } \\
\hline $\begin{array}{l}\text { Píxeles } \\
\text { clasificados como }\end{array}$ & CN & MLD & Nudos & Total píxeles \\
\hline CN & $\mathbf{1 9 2 7}$ & 226 & 3 & 2156 \\
MLD & 5 & $\mathbf{1 0 2 5 3}$ & 203 & 10461 \\
Nudos & 146 & 1607 & $\mathbf{2 3 0}$ & 1983 \\
\hline Total píxeles & 2078 & 12086 & 436 & $\mathbf{1 4 6 0 0}$ \\
Precisión (\%) & $\mathbf{9 2 . 7}$ & 84.8 & 52.8 & $\mathbf{8 5}$ \\
\hline
\end{tabular}


Los resultados obtenidos para los valores de precisión individual y global, luego de aplicar un filtro de mediana de $7 \times 7$ a los mapas temáticos, se presentan en la tabla 2. De acuerdo a esta tabla, valores de precisión de $96.3 \%, 93.2 \%$ y $49.1 \%$ fueron obtenidos para el CN, MLD y nudos, respectivamente. La precisión global fue de $92.3 \%$. Si comparamos estos valores de precisión con los valores presentados en la tabla 1 , se observa que la precisión de detección mejora después de filtrar los mapas temáticos, excepto para la clase nudos la cual disminuyó en un $3.7 \%$ aprox. También se observa que la tendencia en los resultados se mantiene, en el sentido que $\mathrm{CN}$ y nudos presentan el mayor y menor valor de precisión, respectivamente. Por otra parte, y como se esperaba, los errores de omisión para CN y MLD disminuyeron, debido al aumento de los valores de precisión. En este caso, y tal como se observó para los mapas temáticos sin clasificar, los principales errores de omisión están asociados con la clase nudos.

Tabla 2. Matriz de confusión obtenida para los mapas temáticos filtrados.

\begin{tabular}{|ccccc|}
\hline \multicolumn{5}{c|}{ Píxeles en clases verdaderas } \\
\hline $\begin{array}{c}\text { Píxeles } \\
\text { clasificados como }\end{array}$ & CN & MLD & Nudos & Total píxeles \\
\hline CN & $\mathbf{2 0 0 2}$ & 312 & 22 & 2336 \\
MLD & 3 & 11262 & 200 & 11465 \\
Nudos & 73 & 512 & $\mathbf{2 1 4}$ & 799 \\
\hline Total píxeles & 2078 & 12086 & 436 & 14600 \\
Precisión (\%) & $\mathbf{9 6 . 3}$ & 93.2 & 49.1 & $\mathbf{9 2 . 3}$ \\
\hline
\end{tabular}

Esta situación está de acuerdo con los resultados del análisis cualitativo, en el sentido que existe un cierto nivel de superposición del nivel de gris entre estas clases. Aunque la literatura revisada no reporta estudios sobre la identificación del $\mathrm{CN}$ en otras especies de coníferas, los resultados cuantitativos obtenidos de este estudio para MLD y precisión global son comparables con los reportados por otros investigadores. Schmoldt et al. 2000, para las tres especies de latifoliadas evaluadas, reportaron valores de precisión de $94.7 \%$ para MLD y valores entre $90.0 \%$ y $97.0 \%$ para la precisión global. Para pino silvestre Nordmark (2002), reportó un precisión global entre 93 y 95 $\%$. Wei et al. 2008b, para picea negra, reportaron valores de MLD y precisión global de $80.7 \%$ y 92.4 $\%$, respectivamente.

Finalmente, los valores obtenidos para el índice Kappa, a partir de las matrices de confusión de los mapas temáticos y mapas temáticos filtrados, fueron de 0.607 y 0.764 , respectivamente. Estos valores indican, de acuerdo a la escala establecida por Landis y Koch (1977), la existencia de un fuerte grado de conformidad entre los datos de referencia y los resultados del proceso de clasificación usando RNA.

\section{CONCLUSIONES}

Este estudio investigó la posibilidad de identificar y separar el cilindro nudoso de la madera sin defectos y nudos en imágenes TC de pino radiata usando un algoritmo de clasificación basado en redes neuronales. El análisis cualitativo de los mapas temáticos, indicó que el cilindro nudoso puede ser identificado y separado de la madera libre de defectos y de los nudos, debido a la diferencia observada entre los niveles de grises asociados a estas características. Sin embargo, se observó una leve superposición entre el nivel de gris asociado a MLD y nudos, lo cual puede estar asociado a la variación de la densidad y contenido de humedad en el interior de la troza. Los resultados de la evaluación cuantitativa indicaron que el cilindro nudoso puede ser identificado con una precisión de $92.7 \%$. Sin embargo para la precisión global se obtuvo un valor de $85,0 \%$. Después de filtrar los mapas temáticos los valores aumentaron a $96.3 \%$ y $92.3 \%$ para el cilindro nudoso y precisión global. Los valores de índice Kappa obtenidos para los mapas temáticos (0.607) y mapas temáticos filtrados 
(0.764) indicaron que existe un fuerte grado de conformidad entre los datos de referencia y los resultados obtenidos del proceso de clasificación usando redes neuronales. Finalmente, los resultados obtenidos de este estudio sugieren que el algoritmo basado en redes neuronales artificiales presenta un muy buen desempeño en la identificación y segmentación del cilindro nudoso en imágenes TC de trozas de pino radiata.

\section{AGRADECIMIENTOS}

Los autores agradecen al personal del laboratorio de imagenología del Hospital del trabajador por su asistencia durante la etapa de escaneo de las trozas, a la Sra. Linette Salvo, Departamento de Ingeniería en Maderas de la Universidad del Bío-Bío, por el apoyo logístico durante el desarrollo de la parte experimental. Esta investigación fue financiada por el Fondo Nacional de Desarrollo Científico y Tecnológico de Chile, a través del proyecto de iniciación FONDECYT № 11060390.

\section{BIBLIOGRAFIA}

Bhandarkar, S.M.; Faust, T.D.; Tang, M. 1999. CATALOG: a system for detection and rendering of internal log defects using computer tomography. Machine Vision and Application 1999(3):171-190.

Birkeland, R.; Han, W. 1991. Ultrasonic scanning for internal log defects. $4^{\text {th }}$ International Conference on Scanning Technology in the Wood Industry. Burlingame, CA, U.S.A., October 28-29, 1991.

Chang, S.J.; Olson, J.R.; Wang, P.C. 1989. NMR imaging of internal features in wood. Forest Products Journal 39(6):43-49.

Coates, E.R.; Chang, S.J.; Laio, T.W. 1998. A quick defect detection algorithm for magnetic resonance images of hardwood logs. Forest Product Journal 48(10):68-74.

Funt, B.V.; Bryant, E.C.1987. Detection of internal log defects by automatic interpretation of computer tomography images. Forest Products Journal 37(1): 56-62.

Guddanti, S.; Chang S.J. 1998. Detection of internal defects by automatic interpretation of computer tomography. Forest Product Journal 37(1): 56-62.

Hagman, P.O.G. 1993. Automatic quality sorting of Picea abies logs with a gamma ray log scanner. Scandinavian journal of Forests Research 8: 583-590

Jensen, J. R. 1996. Introductory digital image processing: a remote sensing perspective. Prentice Hall, Upper Saddle River, NJ, U.S.A.

Karsulovic, J.T.; Dinator, M.I.; Morales, R. 2002. Nondestructive gamma radiation methods for detection of central rot in logs of Lenga (Nothofagus pumilio). Forest Products Journal 52(2): 87-93.

Karsulovic, J. T.; Dinator, M. I.; Morales, J.; Gaete, V.; Barrios, A. 2005. Determinación del diámetro del cilindro nudoso en trozas podadas de pino radiata (Pinus radiata) mediante atenuación de radiación gamma. Bosque 26(1): 109-122.

Kininmonth, J.A.; Whitehouse, L.J. 1991. Properties and Uses of New Zealand Radiata pine. Volume one-Wood properties. Ian Bryce Printers Ltd., Roturoa, New Zealand.

Landis, J.R.; Koch, G.C. 1977. The measurement of observer agreement of categorical data. Biometrics 33: 159-174. 
Li, P.; Abbot, A. L.; Schmoldt, D.L. 1996. Automated analysis of CT images for the inspection of hardwood logs. Pages 1744-1749 in Proceeding of the IEEE International Conference on Neural Networks, Washington, DC, U.S.A.

Nordmark, U. 2002. Knot identification from CT images of young Pinus sylvestris saw logs using artificial neural networks. Scandinavian Journal Forest Research 17: 72-78.

Nordmark, U. 2003. Models of knot and log geometry of young Pinus sylvestris sawlogs extracted from computed tomographic images. Scandinavian Journal of Forest Research 18:168-175.

Oja, J. and Temnerud. 1999. The appearance of resin pocket in CT images of Norway spruce (Picea abies (L.) Karst.). Holz als Roh-und Werkstoff 57:400-406.

Oja, J. 2000. Evaluation of knot parameters measured automatically in CT-images of Norway spruce (Picea abies (L.) Karst.). Holz als Roh-und Werkstoff 58: 375-379.

PCI 2005. PCI Geomatics, user guide. Volume I. Version 10.1. Richmond Hill, ON, Canada.

Palmer, A.; Montaño, J.J.; Calafat, A. 2000. Predicción del consumo de éxtasis a partir de redes neuronales artificiales. Adicciones 12(1): 29-41.

Rojas, G.; Hernández, R.E.; Condal, A.; Verret, D.; Beauregard, R. 2005. Exploration of the physical properties of internal characteristics of sugar maple logs and relationships with CT images. Wood and Fiber Science 37(4): 591-603.

Rojas, G.; Condal, A.; Beauregard, R.; Hernández, R.E.; Verret, D. 2006. Identification of internal defects of sugar maple logs from CT Images using supervised classification methods. Holz als Roh-und Werkstoff 64 (4): 295-303.

Rojas, G.; Beauregard, R.; Hernández, R.E; Verret, D.; Condal, A. 2007. Effect of moisture content variation on CT image classification to identify internal defects of sugar maple logs. Forest Products Journal 57(4): 38-43.

Rojas, G.; Ortiz, O. 2009. Identificación del cilindro nudoso en imágenes TC de trozas podadas de Pinus radiata utilizando el clasificador de máxima verosimilitud. Maderas. Ciencia y tecnología 11(2): 117-127.

Richards, J.A.; Jia, X. 1999. Remote Sensing Digital Image Analysis - An Introduction. Springer Verlag Berlin Heidelberg.

Rumelhart, D.E.;Hinton, G.E.; Williams, R.J. 1986. Learning internal representations by error propagation. En: Rumelhart y McClelland (Eds.). Parallel distribution processing (pp. 318-362) Cambridge, MA:Mit Press.

Schmoldt D.L.; Li P.; A.L. Abbott. 1995. Log defect recognition using CT-images and neural net classifiers. $2^{\text {nd }}$ International Workshop/Seminar on Scanning Technology and Image Processing on Wood. Skellefteå Sweden, Aug 14-16. pp 77-97

Schmoldt, D.L.; He, J.; Abbott, A.L. 2000. Automated labeling of log features in CT imagery of multiple hardwood species. Wood and Fiber Science 32(3): 287-300. 
Schmoldt, D. L.; He, J.; Abbott, A.L.1998. A comparison of several artificial neural network classifiers for CT images of hardwood logs. Proceedings of SPIE. Sponsored by The Society for Imaging Science and Technology and The International Society for Optical Engineering. San Jose, California. USA. Vol. 3306

Story, M.; Congalton, R.G. 1986. Accuracy assessment: a user's perspective. Photogrammetric Engineering and remote sensing. 54(3): 397-399.

Taylor, F.W.; Wagner, Jr., F.G.; McMillin, C.W. 1984. Locating knots by industrial tomography-A feasibility study. Forest Products Journal 34(5): 42-46.

Wei, Q.; Leblon, B.; Chui, Y. C.; Zhang, S.Y. 2008a. Identification of selected log characteristics from computed tomography images of sugar maple logs using maximum likelihood classifier and textural analysis. Holzforschung 62: 441-447.

Wei, Q.; Chui, Y.C.; Leblon, B.; Zhang, S.Y. 2008b Identification of log characteristics in tomography images using back-propagation neural networks with the resilient back- propagation training algorithm and textural analysis: preliminary results. Wood Fiber Sci 40(4): 620-633

Zhu, D.; Conners, R.W.; Lamb, F.; Araman, P.A. 1991. A computer vision system for locating and identifying internal log defects using CT imagery. Pages 1-13 in Proc. Fourth International Conference on Scanning Technology in the Wood Industry, Burlingame, CA, U.S.A. 
